\title{
IDUS - Instrumental Delivery \& UltraSound. A multi-centre randomised controlled trial of ultrasound assessment of the fetal head position versus standard care as an approach to prevent morbidity at instrumental delivery.
}

Authors:

Meenakshi Ramphul, MRCPI, Research Fellow in Obstetrics, Academic Department of Obstetrics and Gynaecology, Trinity College Dublin, Coombe Women and Infants University Hospital Dublin 8, Ireland email: ramphulm@tcd.ie (corresponding author)

Poh Vei Ooi, MRCOG MRCPI, Research Fellow in Obstetrics, Mid-Western Regional Maternity Hospital, Ennis Road, Limerick, Ireland.

Email: ooiwendee@gmail.com

Gerard Burke, FRCOG, Consultant Obstetrician and Gynaecologist; Honorary Senior Lecturer, University of Limerick, Mid-Western Regional Maternity Hospital, Ennis Road, Limerick, Ireland.

Email: ger_burke@eircom.net

Mairead M Kennelly, MD MRCOG, Consultant Obstetrician and Gynaecologist, Fetomaternal medicine specialist, Senior Lecturer University College Dublin, Coombe Women \& Infant's University Hospital, Dublin 8, Ireland. 
Email: mkennelly@ doctors.org.uk

Soha A.T Said, MRCOG, Consultant Obstetrician and Gynaecologist; Mid-Western Regional Maternity Hospital, Ennis Road, Limerick, Ireland.

Email: sohatohamy@hotmail.com

Alan A. Montgomery, PhD, Reader in Health Services Research, Bristol Randomised Trials Collaboration School of Social and Community Medicine, University of Bristol, Canynge Hall, 39 Whatley Road, Bristol BS8 2PS.

email: alan.a.montgomery@bristol.ac.uk

Deirdre J Murphy, MD FRCOG, Professor of Obstetrics and Head of Department, Academic Department of Obstetrics and Gynecology, Trinity College Dublin, Coombe Women and Infants University Hospital, Dublin 8, Ireland email: murphyd4@tcd.ie (guarantor)

Trial registration number: Current Controlled Trials ISRCTN 72230496

Word count: Abstract: $242 \quad$ Paper: 3140 


\section{Abstract}

Objectives: To determine whether the use of ultrasound can reduce the incidence of incorrect diagnosis of the fetal head position at instrumental delivery and subsequent morbidity.

Design: Two-arm, parallel, randomised trial, conducted from June 2011 to December 2012.

Setting: Two maternity hospitals in the Republic of Ireland.

Sample: 514 nulliparous women at term ( $\geq 37$ weeks' gestation) with singleton cephalic pregnancies, aiming to deliver vaginally were recruited prior to induction of labour or in early labour.

Methods: If instrumental delivery was required, women who had provided written consent were randomised to receive clinical assessment (standard care) or ultrasound scan and ultrasound assessment (ultrasound).

Main outcome: Incorrect diagnosis of the fetal head position.

Results: The incidence of incorrect diagnosis was significantly lower in the ultrasound group than the standard care group $(4 / 257,1.6 \%$ versus $52 / 257,20.2 \%$, odds ratio $0.06,95 \%$ confidence interval $(\mathrm{CI}) 0.02$ to 0.19 , p value $<0.001)$. The decision to delivery interval was similar in both groups (ultrasound mean 13.8 minutes, SD 8.7, versus standard care mean 14.6 minutes, SD 10.1, p value 0.35). The incidence of maternal and neonatal complications, 
failed instrumental delivery and caesarean section was not significantly different between the two groups.

Conclusions: An ultrasound assessment prior to instrumental delivery reduced the incidence of incorrect diagnosis of the fetal head position without delaying delivery but did not prevent morbidity. A more integrated clinical skills-based approach is likely to be required to prevent adverse outcomes at instrumental delivery.

Trial registration number: Current Controlled Trials ISRCTN 72230496

Key words: fetal head position; second stage of labour; intrapartum ultrasound, randomised controlled trial 


\section{Introduction}

Most first time mothers are aiming for an uncomplicated vaginal birth but many experience complications in labour, resulting in instrumental delivery or caesarean section. In these circumstances, the obstetrician is aiming to assist by the safest means possible, cognisant that the mode of delivery and related morbidities will have implications for future deliveries. ${ }^{1,2}$ Operative delivery rates vary greatly between operators, institutions and countries, particularly for first time mothers. In the United States of America, the overall caesarean section rate was $32.8 \%$ in 2010 and the instrumental delivery rate $3.6 \% .{ }^{3}$ In the United Kingdom and Ireland, caesarean section rates vary between 20 and 30\% with instrumental delivery rates between 12 and $17 \% .{ }^{4,5}$ Rates of instrumental delivery are highest among first time mothers accounting for up to $30 \%$ of births. The caesarean section rate continues to rise globally. It has been suggested that greater skill in instrumental delivery could reduce caesarean section rates, particularly complex caesareans performed in the second stage of labour. ${ }^{6,7}$

Instrumental delivery is associated with an increased risk of maternal and neonatal morbidity but with skilled care the risks are low. ${ }^{2,8-10}$ Correct diagnosis of the fetal head position is a pre-requisite for safe instrumental delivery. ${ }^{11,12}$ Diagnosis of a fetal malposition will influence the level of skill required of the operator, the choice of instrument, the place of delivery and the mode of delivery. Serious maternal and neonatal trauma is associated with excessive pulls, sequential use of instruments, and caesarean section after a failed attempt at instrumental delivery. ${ }^{2}$ Failure to identify a malposition (especially an occipito-posterior position) is one of the factors that increases the likelihood of failed instrumental delivery and neonatal trauma. ${ }^{13-19}$ 
The fetal head position is diagnosed on vaginal examination by delineating the suture lines of the fetal skull and fontanelles. However, accurate clinical diagnosis can be unreliable, varying between 20 and $75 \% .^{20-29}$ The use of abdominal ultrasound to enhance the diagnosis of the fetal head position has been described in a number of small studies but only two have evaluated the role of ultrasound at instrumental delivery. ${ }^{21,26,27,29-31}$

We aimed to compare ultrasound assessment of the fetal head position prior to instrumental delivery with standard care to determine whether the use of ultrasound can reduce the incidence of incorrect diagnosis of the fetal head position. We postulated that a routine ultrasound scan in addition to clinical examination would reduce the incidence of incorrect diagnosis of the fetal head position and delivery-related maternal and neonatal morbidity.

\section{METHODS}

The Instrumental Delivery and UltraSound (IDUS) trial was a two arm, parallel, randomised controlled trial.

\section{Population}

We recruited women from two university teaching hospitals in Ireland with a combined annual birth rate of 13,500 deliveries (40\% nulliparous; overall instrumental delivery rate $18 \% ; 33 \%$ for nulliparous women). In these units, instrumental deliveries are carried out by obstetricians of varying experience with a consultant supervising the labour ward onsite during the day and offsite at night. Nulliparous women at term (at least 37 completed weeks' gestation) with singleton cephalic pregnancies, aiming to deliver vaginally were eligible to participate. We excluded women under 18 years of age, with limited understanding of English, or with a contraindication to instrumental delivery. Eligible women provided written 
consent prior to induction of labour or in early labour. Obstetricians could exclude women at their discretion where there was immediate urgency due to fetal compromise.

\section{Intervention and comparison}

We compared clinical and ultrasound assessment of the fetal head position with standard care (clinical examination alone).

\section{Outcome measures}

Primary outcome

The primary outcome measure was incorrect diagnosis of the fetal head position. We debated whether to use maternal and/or neonatal morbidity as the primary outcome but chose incorrect diagnosis of the fetal head position as this relates directly to the trial intervention, rather than morbidity, which may result from many factors on the clinical pathway from decision for instrumental delivery to completed delivery. The primary outcome was established in two ways. If the position of the fetal head before delivery was classified as OA and delivered OP, the diagnosis was considered incorrect. Furthermore, the midwife or neonatologist who attended the delivery examined the neonate and recorded the markings of the instrument on a drawing of the head and face. (Appendix 2) The recorded markings were used to indicate misplacement of the instrument at a distance from the flexion point (vacuum) or over the face (forceps), the diagnosis being considered incorrect if the markings were more than $45^{\circ}$ from the documented fetal head position. For example, if the recorded position prior to instrumental delivery was OA and the instrument placement suggested an OT or OP position, the diagnosis was considered incorrect. The primary outcome was validated independently by a single investigator (DJM) who was not involved in scanning and who was blinded to trial allocation. 
Secondary outcomes

Maternal morbidity outcomes included extensive perineal tearing involving the anal sphincter (third or fourth degree tears), postpartum haemorrhage (estimated blood loss greater than $500 \mathrm{mls}$ ), shoulder dystocia, and length of postnatal hospital stay (prolonged if more than three days' duration). Neonatal morbidity outcomes included trauma, fetal acidosis (defined as arterial $\mathrm{pH}$ below 7.10 and base excess greater than $-12.0 \mathrm{mmol} / \mathrm{l}$ ) and admission to the neonatal intensive care unit (NICU). Neonatal trauma included cephalhaematoma, intracranial haemorrhage, retinal haemorrhage, facial nerve palsy, brachial plexus injury and fractures. Mothers and neonates were followed up for complications until hospital discharge.

Procedural outcomes were recorded in terms of the decision to delivery interval (the time from making the decision to intervene until delivery of the infant), place of delivery, need for senior obstetric support, transfer to theatre, use of sequential instruments (more than one instrument), failure of instrumental delivery followed by caesarean section, or immediate caesarean section.

\section{Trial procedures}

After the decision to perform an instrumental delivery had been made, eligible women who had provided written informed consent were randomly assigned to either clinical examination and an ultrasound scan or clinical examination alone (standard care). Women allocated to standard care were assessed by abdominal and vaginal examination according to the guidelines of the Royal College of Obstetricians and Gynaecologists (RCOG). ${ }^{32}$ Following clinical examination, the fetal head position was recorded by way of a cross on a data sheet depicting a circle, like a clock, divided into 8 sections, each of 45 degrees. (Appendix 1) The 
position was classified as OA for direct occipito-anterior, ROA and LOA for right and left occipito-anterior respectively; OP for direct occipito-posterior, ROP and LOP for right and left occipito-posterior; ROT and LOT for right and left occipito- transverse respectively. The obstetrician then proceeded to instrumental delivery as usual.

Women in the ultrasound group were managed in the same way. In addition the researcher performed an ultrasound scan to assess the position of the fetal head and spine. The obstetrician was provided with the ultrasound findings and used this information together with the clinical findings to define the position prior to instrumental delivery. Where there was discordance between the clinical and scan findings, the researcher recorded whether or not the ultrasound finding was accepted.

Two researchers (MR and PVO) were trained in ultrasound assessment by a sub-specialist in fetal and maternal medicine before the start of the trial. ${ }^{29}$ Image-directed pulsed Doppler equipment (Sonosite Titan) with a multifrequency sector array transabdominal transducer, and a $3.5 \mathrm{MHz}$ sector ultrasound probe was used for all ultrasound scans. The ultrasound probe was placed transversely over the maternal abdomen to identify the fetal spine, and then moved towards the pubic region to obtain a view of the fetal head. The landmarks of the fetal head used to identify and classify the position were as follows: midline cerebral echo, falx cerebri, thalamus, the orbits and the nuchal region. (Figure 1)

\section{Randomisation}

Women were assigned to study group in a 1:1 ratio using a secure web-based central randomisation service ensuring concealment of allocation. The allocation sequence was 
computer-generated, stratified by centre, and used random permuted blocks of sizes of 4,8 and 12 .

\section{Study oversight}

An independent trial steering committee (TSC) was set up to provide oversight of the study. We received institutional ethics approval and written informed consent from each woman. The study was conducted in accordance with the protocol. ${ }^{32}$ The TSC advised us that a separate data monitoring committee was not required as serious adverse events in this trial were likely to be inherent complications of the procedure (instrumental delivery) and unlikely to be related to the intervention (ultrasound).

\section{Statistical analysis}

The incidence of incorrect diagnosis of fetal head position for the standard care group was estimated as $20 \%$ based on the published literature and we sought to detect an absolute between-group difference of $10 \%$ which we regarded as sufficient to change practice. ${ }^{21-30}$ With $80 \%$ power and 5\% two-sided alpha, a total sample size of 450 for analysis was required. We inflated the target sample to 500 to allow for up to $10 \%$ non-collection of primary outcome data, for example due to spontaneous vaginal deliveries after randomisation but before diagnosis.

We used descriptive statistics to assess the comparability of the trial groups at baseline. All between-group comparisons were conducted on an intention to treat basis without imputation - that is, all participants were analysed according to their randomised groups, and complete data collection for all outcomes meant that no imputation of missing data occurred. We used appropriate, that is logistic or linear, multivariable regression models to estimate between- 
group differences in the primary and secondary outcomes, adjusted for centre as a stratification variable. In sensitivity analyses of the primary outcome, we investigated the effect of further adjustment for any variables that were imbalanced at baseline, and of clustering by operator by using mixed effects regression. However the latter analysis had to be restricted to participants for whom the operator was known as this was recorded in only one of the two study centres. In pre-planned subgroup analyses we investigated whether any effect of ultrasound on the primary outcome differed according to study centre or operator experience by including appropriate interaction terms in the regression models. All statistical analyses were conducted using SPSS 18 and Stata 12.

\section{RESULTS}

\section{Study population}

Between June 2011 and December 2012, we enrolled and randomised a total of 514 women, 257 to ultrasound and 257 to standard care. Figure 2 shows the participant flow.

\section{Descriptive statistics}

Baseline characteristics were similar between the two groups with small differences in pathological cardiotocograph (CTG) and senior obstetrician. (Table 1) There were eleven spontaneous vaginal deliveries in the ultrasound group and ten in the standard care group, after randomisation had occurred.

\section{Primary outcome}

The incidence of incorrect diagnosis of the fetal head position was significantly lower in the ultrasound group compared to the standard care group (ultrasound 4/257, 1.6\%; standard care $52 / 257,20.2 \%$, adjusted odds ratio (OR) $0.06,95 \%$ confidence interval (CI) 0.02 to $0.19, \mathrm{p}$ 
value $<0.001$ ). (Table 2 ) The results did not change when the following variables were taken into account: centre, pathological CTG and senior obstetrician (adjusted OR 0.06, 95\% CI 0.02 to 0.16 , $\mathrm{p}$ value $<0.001)$ Further analyses which investigated whether the effect of the intervention differed according to individual operator clustering were not significant.

\section{Subgroup analyses}

There was a significant interaction seen between ultrasound and study centre. The benefit of ultrasound to reduce the incorrect diagnosis was greater in the first centre (OR 0.03 in first centre versus OR 0.41 in second centre; interaction co-efficient $13,95 \%$ CI 1.5 to 120$)$. This finding is probably due to the overall lower rates of incorrect diagnoses in the Limerick centre where more senior obstetricians performed the delivery and where vacuum deliveries were predominantly performed in some cases making incorrect diagnosis more difficult to establish.

\section{Secondary outcomes}

The incidence of maternal and neonatal complications was not significantly different between the two groups. (Table 3) There were three neonates who required neurological follow-up at discharge in both groups. There was one neonatal death in the ultrasound group from congenital cardiac anomalies. The mean time taken to perform the ultrasound scan was thirty seconds (range 5 to 120 seconds, standard deviation 22 seconds). (Table 4) The decision to delivery interval was not longer in the ultrasound group (ultrasound mean 13.8 minutes, SD 8.7 versus standard care mean 14.6 minutes, SD 10.1, difference in means $-0.78,95 \%$ CI $0.85-2.42, \mathrm{p} 0.35)$. The choice of primary instrument used for delivery was similar in the ultrasound and standard care groups. There was no significant difference in the number of sequential instruments used or caesarean sections after failed instrumental delivery. (Table 4) 
There was weak evidence of an association in the ultrasound group with less immediate caesarean sections $(2 / 257,0.8 \%$ versus $8 / 257,3.1 \%$; odds ratio $0.24,95 \%$ confidence interval 0.05-1.16, p 0.07). Overall, the ultrasound scan diagnosis was accepted in 242/257 (94.2\%) cases and not accepted in 9/257 (2.5\%) cases (unrecorded in 6 cases). There was significant maternal and neonatal morbidity in one case where the ultrasound diagnosis of a fetal malposition was not accepted.

\section{DISCUSSION}

\section{Main findings}

The results of this multicentre randomised trial show that an ultrasound assessment prior to instrumental delivery reduces the incidence of incorrect diagnosis of the fetal head position without delaying the delivery. However, enhanced diagnosis of the fetal head position did not reduce the incidence of maternal or neonatal complications nor were there significant differences in instrument choice or mode of delivery between the two groups.

\section{Strengths and limitations}

The strengths of our trial include its large size, multicentre design and the high compliance with group allocation after randomization. The study population included a range of nulliparous women with varying body mass index, different types of anaesthesia and the full range of indications for instrumental delivery, including fetal distress. Operators at all levels of experience were included. The results are generalisable to other centres where instrumental deliveries are performed.

The limitations of this trial should be considered. While the researchers attempted to capture a variety of deliveries throughout the day and night, the majority of women were recruited 
during 'regular' working hours. We may have missed deliveries at night time where less experienced obstetricians managed complex cases with indirect supervision. Furthermore, it was challenging in some cases to differentiate between incorrect diagnosis of the fetal head position and suboptimal instrument placement, particularly in cases of vacuum delivery. It is possible that the rate of incorrect diagnosis was even higher in the standard care group. We had considered an alternative study design where we would perform an ultrasound scan on every woman and randomise to reveal or conceal the findings for fetal head position. This was deemed unethical, as concealing a fetal malposition from an inexperienced operator could result in significant morbidity, and flawed in terms of equipoise, as ultrasound would have been assumed to be more accurate than clinical examination.Although the study protocol was adhered to in both centres, we provided no direction on choice of instrument and there was a greater preference for vacuum delivery in centre two. In cases of uncertainty we gave the operator the benefit of the doubt and classified the position as "correct". This is likely to account for the difference in the incidence of incorrect fetal head position between the two centres.

\section{Interpretation}

The incidence of incorrect diagnosis of the fetal head position by clinical examination alone was $20 \%$ which is at the lower end of the published literature. ${ }^{20-29}$ Most studies have compared ultrasound assessment and clinical examination earlier in labour rather than immediately before instrumental delivery. There may be less care taken with clinical examination earlier in labour given that an accurate diagnosis is less critical to safety, unlike with instrumental delivery. Two small scale studies had findings similar to ours. ${ }^{21,31}$ A cohort study of 64 women reported an incorrect diagnosis rate of $27 \%$ for vaginal examination compared to ultrasound, with errors more likely with OP positions. ${ }^{21}$ A randomised trial of 
fifty women undergoing vacuum extraction for prolonged second stage reported that cup placement was closer to the flexion point and therefore more optimal in the group assigned to ultrasound compared to vaginal examination only. ${ }^{31}$

The decision to delivery interval is an important consideration for instrumental delivery. ${ }^{34}$ It was reassuring that the addition of an ultrasound scan as part of the assessment did not introduce a delay in delivery. Clinical guidelines highlight the greater risk of failed instrumental delivery with a fetal malposition and recommend that recourse to caesarean section is available by transferring the patient to an operating theatre. ${ }^{11}$ We were surprised that the enhanced diagnosis of fetal malpositions in the ultrasound group did not appear to influence management decisions and equally that the higher rate of incorrect diagnoses in the standard care group did not affect the rate of sequential instruments or failed instrumental deliveries. These findings reflect the complexity of instrumental delivery. There are other factors such as engagement, station of the presenting part, fetal size, and maternal pelvic dimensions that may contribute to procedural decisions. ${ }^{13}$ One potential explanation is that ultrasound enhanced the diagnosis of fetal malpositions but not the operators ability to deal with it. Given that enhanced diagnosis of the fetal head position had little impact on the management decisions made by operators it is perhaps unsurprising that morbidity rates were similar in both groups. Of note, serious maternal and neonatal complications in this study were low and comparable to previous published data. ${ }^{2,13}$ Future trials should incorporate the wider dimensions of clinical assessment and decision-making, as ultrasound guided assessment of the fetal head position, while necessary, is insufficient in itself to prevent morbidity at instrumental delivery.

\section{Clinical implications}


The use of ultrasound on the labour ward is increasing with ready access to portable ultrasound equipment. ${ }^{33}$ Our previous work demonstrated that abdominal ultrasound is acceptable to women in labour and to clinicians looking after them. ${ }^{29}$ Furthermore, obstetric trainees can acquire the skills to perform an accurate ultrasound diagnosis of the fetal head position in labour within a short timeframe. ${ }^{29,30}$ Given that knowledge of the fetal head position is a prerequisite for safe instrumental delivery, our findings suggest that ultrasound has an important role to play in getting this element of assessment right. The next stage is to establish how to translate enhanced assessment into better clinical decision-making. Among the secondary outcomes, the lower rate of transfers to theatre and immediate caesarean sections in the ultrasound group warrants further evaluation in a large appropriately powered trial but the potential benefits would need to be balanced with a higher risk of excessive pulls (>3) at instrumental delivery.

\section{Conclusions}

Our findings support the use of ultrasound prior to instrumental delivery to identify the fetal head position but also demonstrate that an imaging approach in isolation will not reduce morbidity. A more integrated clinical skills-based approach is likely to be required to enhance the safety of instrumental delivery, particularly when a fetal malposition has been identified. 


\section{Conflicts of interest}

All authors have no financial connections with companies that may have an interest in the submitted work, and no non-financial interests that may be relevant to the article.

\section{Acknowledgements}

We thank all the women who took part in the IDUS study; the labour ward midwives, obstetricians and neonatologists who supported recruitment for the study; the recruitment advisors Sharon Sheehan and Maureen Macleod; the members of the trial steering committee Geraldine Gaffney (chair), Andrew Weeks, Rebecca Cannings-John (independent statistician); Chris Fitzpatrick, sponsor on behalf of the Coombe Women \& Infants University Hospital; the Midwestern Regional Hospital Limerick; Piaras Fahey and Clare Dunney for assistance with the database; Tom Fahey for reviewing the manuscript; and our funders the Health Research Board of Ireland.

\section{Contribution to authorship}

DJ Murphy and G Burke had the original idea for the study. DJ Murphy, G Burke, M Kennelly, A Montgomery and M Ramphul designed the trial. M Ramphul and A Montgomery performed the analyses. M Ramphul and DJ Murphy drafted the paper which was revised by all authors. DJ Murphy is the guarantor.

\section{Details of Ethics approval}

We received ethical approval from the Ethics Research Committee in the Coombe Women \& Infants University Hospital on the $5^{\text {th }}$ October 2010 and from the Ethics Research Committee in the Mid-Western Regional Maternity Hospital, Limerick on the $9^{\text {th }}$ August 2011. 


\section{Funding source}

The trial was funded by the Health Research Board of Ireland (POR/2010/55). The funding source had no role in the trial design, writing of the report, or the decision to submit the paper for publication. 


\section{References}

1. Patel RR, Murphy DJ. Forceps delivery in modern obstetric practice. $B M J$ 2004;328(7451):1302-5.

2. Murphy DJ, Liebling RE, Patel R, Verity L, Swingler R. Cohort study of operative delivery in the second stage of labour and standard of obstetric care. Br J Obstet Gynaecol 2003;110:610-5.

3. Martin J, Hamilton B, Ventura S, Osterman M, Wilson E, Matthews T. Births: Final data for 2010. Natl Vital Stat Rep 2012;61(1).

4. BirthChoiceUK [Internet]. United Kingdom; 2010. Available from:

http://www.birthchoiceuk.com/Professionals/Frame.htm (last accessed: 15 February 2013)

5. Consumer guide to maternity services in Ireland [Internet]. Ireland; 2010. Available from: http://www.bump2babe.ie/column/N/statistics/ (last accessed: 15 February 2013)

6. Evaluation of Caesarean Delivery. Washington, DC: American College of Obstetricians and Gynecologists, 2000.

7. Spencer, C, Murphy DJ, Bewley S. Caesarean section in the second stage of labour. BMJ 2006;333:613-4. 
8. Towner D, Castro MA, Eby-Wilkens E, Gilbert WM. Effect of mode of delivery in nulliparous women on neonatal intracranial injury. N Engl J Med 1999;341(23):1709-14.

9. Whitby EH, Griffiths PD, Rutter S, Smith MF, Sprigg A, Ohadike P, et al. Frequency and natural history of subdural haemorrhages in babies and relation to obstetric factors. Lancet 2004;363(9412):846-51.

10. Demissie K, Rhoads GG, Smulian JC, Balasubramanian BA, Gandhi K, Joseph KS, et al. Operative vaginal delivery and neonatal and infant adverse outcomes: population based retrospective analysis. BMJ 2004;329(7456):24-9.

11. Royal College of Obstetricians and Gynaecologists. Operative vaginal delivery. Greentop guideline No. 26. January 2011. Bahl R, Strachan B, Murphy DJ.

12. American College of Obstetricians and Gynecologists. ACOG. Practice Bulletin 17. Operative vaginal delivery. Washington DC: 2000.

13. Murphy DJ, Macleod M, Bahl R, Strachan B. A cohort study of maternal and neonatal morbidity in relation to use of sequential instruments at operative vaginal delivery. Eur $J$ Obstet Gynecol Reprod Biol 2011;156(1):41-5.

14. Ebulue V, Vadalkar J, Cely S, Dopwell F, Yoong W. Fear of failure: are we doing too many trials of instrumental delivery in theatre? Acta Obstet Gynecol Scand 2008;87(11):1234-8. 
15. Murphy DJ, Liebling R, Verity L, Swingler R, Patel R. Cohort study of the early maternal and neonatal morbidity associated with operative delivery in the second stage of labour. Lancet 2001;358:1203-7.

16. Ben-Haroush A, Melamed N, Kaplan B, Yogev Y. Predictors of failed operative vaginal delivery: a single-center experience. Am J Obstet Gynecol 2007;197(3):308.e1-5.

17. Ponkey SE, Cohen AP, Heffner LJ, Lieberman E. Persistent fetal occiput posterior position: Obstetric outcomes. Obstet Gynecol 2003;101(5):915-20.

18. To WW, Li IC. Occipital posterior and occipital transverse positions: reappraisal of the obstetric risks. Aust N Z J Obstet Gynaecol 2000;40(3):275-9.

19. Cheng YW, Shaffer BL, Caughey AB. Associated factors and outcomes of persistent occiput posterior position: A retrospective cohort study from 1976 to 2001. J Matern Fetal Neonatal Med 2006;19(9):563-8.

20. Akmal S, Tsoi E, Kametas N, Howard R, Nicolaides KH. Intrapartum sonography to determine fetal head position. J Matern Fetal Neonatal Med 2002;12(3):172-7.

21. Akmal S, Kametas N, Tsoi E, Hargreaves C, Nicolaides KH. Comparison of transvaginal digital examination with intrapartum sonography to determine fetal head position before instrumental delivery. Ultrasound Obstet Gynecol 2003;21(5):437-40. 
22. Dupuis O, Ruimark S, Corinne D, Simone T, Andre D, Rene-Charles R. Fetal head position during the second stage of labor: Comparison of digital vaginal examination and transabdominal ultrasonographic examination. Eur J Obstet Gynecol Reprod Biol 2005;123(2):193-7.

23. Souka AP, Haritos T, Basayiannis K, Noikokyri N, Antsaklis A. Intrapartum ultrasound for the examination of the fetal head position in normal and obstructed labor. J Matern Fetal Neonatal Med 2003;13(1):59-63.

24. Sherer DM, Miodovnik M, Bradley KS, Langer O. Intrapartum fetal head position I: Comparison between transvaginal digital examination and transabdominal ultrasound assessment during the active stage of labor. Ultrasound Obstet Gynecol 2002;19(3):258-63.

25. Sherer DM, Miodovnik M, Bradley KS, Langer O. Intrapartum fetal head position II: Comparison between transvaginal digital examination and transabdominal ultrasound assessment during the second stage of labor. Ultrasound Obstet Gynecol 2002;19(3):264-8.

26. Kreiser D, Schiff E, Lipitz S, Kayam A, Avraham A, Achiron R. Determination of fetal occiput position by ultrasound during the second stage of labor. J Matern Fetal Neonatal Med 2001;10(4):283-6.

27. Zahalka N, Sadan O, Malinger G, Liberati M, Boaz M, Glezerman M, et al. Comparison of transvaginal sonography with digital examination and transabdominal sonography for the determination of fetal head position in the second stage of labor. Am J Obstet Gynecol 2005;193(2):381-6. 
28. Chou R, Kreiser D, Taslimi M, Druzin M, El-Sayed Y. Vaginal versus ultrasound examination of fetal occiput position during the second stage of labor. Am J Obstet Gynecol 2004;191:521-4.

29. Ramphul M, Murphy DJ. Establishing the accuracy and acceptability of abdominal ultrasound to define the fetal head position in the second stage of labour: a validation study. Eur J Obstet Gynecol Reprod Biol 2012; 164(1):35-9.

30. Rozenberg P, Porcher R, Salomon LJ, Boirot F, Morin C, Ville Y. Comparison of the learning curves of digital examination and transabdominal sonography for the determination of fetal head position during labor. Ultrasound Obstet Gynecol 2008;31(3):332-7.

31. Wong GY, Mok YM, Wong SF. Transabdominal ultrasound assessment of the fetal head and the accuracy of vacuum cup application. Int J Gynaecol Obstet 2007;98(2):120-3.

32. Murphy DJ, Burke G, Montgomery AA, Ramphul M. Study protocol. IDUS Instrumental delivery \& ultrasound: a multi-centre randomised controlled trial of ultrasound assessment of the fetal head position versus standard care as an approach to prevent morbidity at instrumental delivery. BMC Pregnancy Childbirth 2012;12:95. doi: 10.1186/1471-2393-12-95.

33. Ramphul M, Murphy DJ. The role of ultrasound on the labour ward. Expert Rev Obstet Gynecol 2012; 7 (6): 615- 25. 
34. Murphy DJ, Koh DM. Cohort study of the decision to delivery interval and neonatal outcome for 'emergency' operative vaginal delivery. Am J Obstet Gynecol 2007;196:145.e1-7. 